\title{
TRANSFERABLE UTILITY DISTRIBUTION ALGORITHM FOR MULTICRITERIA CONTROL IN STRONGLY COUPLED SYSTEM WITH PRIORITIES
}

\author{
M.I. Geraskin \\ Samara National Research University, Samara, Russia
}

\begin{abstract}
The problem of multicriteria control on the basis of aggregate utility maximizing is considered. The system utility distribution algorithm in case of unequal criteria set by priority vector, has been developed for strongly coupled technical and organized systems with transferable utility. The Pareto efficiency is proved for the distribution generated on the algorithm basis.
\end{abstract}

Keywords: multicriteria control, aggregate utility, strongly coupled system, transferable utility, distribution algorithm, criteria priorities

Citation: Geraskin MI. Transferable utility distribution algorithm for multicriteria control in strongly coupled system with priorities. CEUR Workshop Proceedings, 2016; 1638: 542-551. DOI: 10.18287/1613-0073-2016-1638-542551

\section{Introduction}

For technical and organizational systems there are now two ways to solve the problem of multicriteria optimal control, efficiency of which is evaluated with the help of several criteria. The first way is based on the Pareto efficiency criterion [1] with settingup of a corresponding set. Another way is Neumann-Morgenstern expected utility maximization [2] in one form or another [3-6]. The latter approach ensures the uniqueness of the solution of the problem. However, its use is justified in case the strongly coupled system model is adequate [7] for the particular group of criteria. The system is regarded as strongly coupled in case the criteria of its elements are compatible with the axioms [8] of preference (reflexivity, connection, transitivity, etc.). It allows for the criteria vector the reduction towards the scalar problem of aggregate utility optimization. Solution of this problem is Pareto-efficient [10] in case it corresponds to the minimax criterion [9]. Utility aggregation may be based on physical and technical properties of systems. In particular, in the terminal control problems [11] deviations from the phase coordinates target values are aggregatively set out in the form of a metric (distance) in the criteria hyperspace. In the problems of multicriteria statistical estimation [12] the moments of random variables are set out in the from of the variation coefficient. In general, the criteria aggregation is based on the properties 
of the system integrity and hierarchal pattern [13], for example, interrelated technologies implementing systems. Due to the production process uniformity the elements of criteria are reduced to the aggregated criterion of the system.

The special case of a strongly coupled system is the transferable utility system with criteria expressed in a single measuring element, for example, in case of homogeneous technologies [14] performance optimization. In general any system of criteria may be formally reduced to the transferable system by means of setting out [9] criteria in a non-dimensional form by means of corresponding normalization. Transferable utility allows for the system elements criteria vector values redistribution (transfer), corresponding to a certain Pareto-efficient control. Consequently, the problem of multicriteria control is reduced to the problem of optimal utility distribution between the system elements on the basis of the aggregated criterion. Solution of the latter problem will also be Pareto-efficient.

Transferable utility distribution algorithms are intended for the systems with elements having equisignificant criteria (of anonymous elements). In this case the aggregation process doesn't take the criteria priorities into account. In particular, Pareto-efficiency is justified [15] in case of an algorithm that establishes the minimum between the element optimum and average non-distributed utility of the system. In case the system elements criteria have different priorities, the distribution algorithms are reduced to the median multicriteria choice [16]. However, in a general Pareto- inefficient case, mechanisms of anonymous symmetric coalitions [17] have been developed. In particular cases these mechanisms are Pareto-efficient. We shall hereinafter consider the problem of development of a Pareto-efficient algorithm of transferable utility distribution between elements with priorities on the basis of the results [14], obtained for anonymous elements.

\section{Problem definition}

Let us consider the system with the elements efficiency determined by the criteria vector $f=\left\{f_{k}(u), k \in K\right\}$. Let the scalar optimum $u_{k}^{0}$ for the criteria of k-element be determined on the basis of the condition

$u_{k}^{0}=\underset{u_{k} \in U_{k}}{\operatorname{Arg} \max } f_{k}(u), f_{k}^{0}=f_{k}\left(u_{k}^{0}\right), k \in K$,

where $u$ - control, $\mathrm{U}$ - feasible region, $f_{k}(u), k \in K$ - k-element efficiency criterion.

Let us form the criteria minimums vector, required for further normalization, from the values obtained in case of scalar optimums of other elements:

$f_{k}^{\min }=\min _{j \in K \backslash k} f_{k}\left(u_{j}^{0}\right), k \in K$.

Subject to (1), (2) the range of criteria values variation equals to $f_{k}\left(u_{k}\right) \in\left[f_{k}^{0}, f_{k}^{\text {nin }}\right]$. 
Let us introduce the hypothesis of benevolence [7], in accordance to which the elements of a strongly coupled system maximize the utility of the whole system. Control is determined on the basis of the maximum value of the sum of criteria in case of optimums of elements. In this context the unique solution of a multicriteria problem is formed. This solution is Pareto-efficient according to the Herneyer's theorem [1,9]. Let us denote this solution by $u^{*}$ and define it as follows:

$u^{*}=\operatorname{Arg} \max _{k \in K} \sum_{j \in K}\left[\max _{u_{k} \in U_{k}} f_{j}\left(u_{k}\right)\right], f_{k}^{*}=f_{k}\left(u^{*}\right), k \in K$,

where $f_{k}^{*}$-k-criterion value in case of the strongly coupled system optimum.

Let us define the strongly coupled system maximum aggregate utility $F\left(u^{*}\right)$ in case of control (3) as exceeding of criteria optimums over their minimum values:

$F\left(u^{*}\right)=\sum_{k \in K}\left(f_{k}^{*}-f_{k}^{\text {min }}\right)$.

Let us introduce the system utility distribution vector $X=\left\{x_{k}, k \in K\right\}$, belonging to the acceptable set

$$
F_{X}=\left\{X=\left\{x_{k}, k \in K\right\}: \sum_{k \in K} x_{k} \leq F\left(u^{*}\right), X \in R_{+}^{k}\right\} .
$$

Let us denote the criteria vector, formed as the result of aggregate utility distribution $f_{k}\left(x_{k}\right)$, and, with account taken of (4), let us define it as follows:

$f_{k}\left(x_{k}\right)=f_{k}^{\text {min }}+x_{k}, k \in K$.

Criteria functions (5) constitute the efficiency criteria of utility distribution between elements in case of Pareto-efficient control (3). Let us formulate the multicriteria problem of optimal utility distribution as follows:

$$
X^{*}=\underset{X \in F_{X}}{\operatorname{Arg} \max }\left\{f_{k}\left(x_{k}\right), k \in K\right\} \text {. }
$$

Let us introduce the criteria priorities vector $\gamma_{k}, k \in K$, the components of which determine their relative significance within the framework of the system aggregate utility. As hereinafter the problem (6) is formulated as minimization of criteria deviations from their scalar maximums, it is expedient to pass on to inverse priorities $\bar{\gamma}_{k}=1-\gamma_{k}, k \in K$, that characterize the relevant significance of other criteria in comparison with this particular criteria. Let us define the vector of inverse priorities as follows

$$
\Gamma=\left\{\gamma_{k} \in R_{+}, k \in K\right\}, \sum_{k \in K} \gamma_{k}=1 .
$$


As it follows from (1), (3) that deviations of criteria (5) from scalar maximums (1) are negative, let us define the normalized values of criteria $\tilde{f}_{k}\left(x_{k}, \bar{\gamma}_{k}\right)$ as squared relevant deviations of criteria (5) and (1) with account taken of inverse priorities:

$\tilde{f}_{k}\left(x_{k}, \gamma_{k}\right)=\left(\frac{\bar{\gamma}_{k} f_{k}\left(x_{k}\right)-f_{k}^{0}}{\bar{\gamma}_{k} f_{k}^{\min }-f_{k}^{0}}\right)^{2}, k \in K$.

Firstly, normalization (8) reduces criteria to the range $\tilde{f}_{k}\left(x_{k}, \bar{\gamma}_{k}\right) \in[0,1]$ that makes it possible to operate on the criteria (8) as transferable ones; secondly, it prescribes the utility transitivity $\tilde{f}\left(x_{k}, \bar{\gamma}_{k}\right) \leq \tilde{f}\left(x_{i}, \bar{\gamma}_{k}\right) \forall \gamma_{k} \geq \gamma_{i}, k \in K \backslash i$ in respect of which higher priority criteria have lesser utility loss.

Let us consider a strongly coupled system, in which control is defined according to (3). It also ensures utility transferability with the help of (8). It allows for [7] the reduction of the multicriteria problem (6) towards the scalar optimization problem by means of introduction of an aggregated utility function in the form of the relative departures product (8):

$$
X^{*}=\underset{X \in F_{X}}{\operatorname{Arg} \min } \prod_{k \in K} \tilde{f}_{k}\left(x_{k}, \bar{\gamma}_{k}\right) .
$$

Criterion in the problem (9) constitutes an aggregated function of the system utility. Moreover, as it is in a normalized form (8) that partial criteria components form part of (9), the effect distribution that satisfies (9) provides [9] with the maximum efficient solution of the multicriteria problem (6) in respect of minimax

$$
\tilde{f}^{*}\left(X^{*}\right)=\min _{X \in F_{X}} \max _{k \in K} \tilde{f}_{k}\left(x_{k}, \bar{\gamma}_{k}\right),
$$

that corresponds, as it is shown in [10], to Pareto efficiency.

\section{Utility distribution algorithm}

Let us formulate the system (4) utility distribution that is optimal in respect of criteria (9) in the form of the following assertion: distribution

$$
x_{k}^{*}=\frac{1}{1+\sum_{i \in K \backslash k} \frac{\bar{\gamma}_{k}}{\bar{\gamma}_{i}}}\left(F\left(u^{*}\right)+\sum_{i \in K \backslash k} f_{i}^{*}+\sum_{i \in K \backslash k} \frac{f_{0 k}-f_{0 i}-\bar{\gamma}_{k} f_{i 0 i}^{*}}{\bar{\gamma}_{i}}\right), k \in K
$$

is the solution of the problem (9).

Proof: differentiating Lagrange function, written down for the problem (9) with consideration of (8) 


$$
L=\prod_{k \in K} \tilde{f}_{k}\left(x_{k}, \bar{\gamma}_{k}\right)+\lambda\left(F-\sum_{k \in K} x_{k}\right)=\prod_{k \in K}\left(\frac{\bar{\gamma}_{k} f_{k}\left(x_{k}\right)-f_{k}^{0}}{\bar{\gamma}_{k} f_{k}^{\text {nin }}-f_{k}^{0}}\right)^{2}+\lambda\left(F-\sum_{k \in K} x_{k}\right),
$$

we arrive at the system of necessary conditions of optimality

$$
\begin{aligned}
& L_{x_{k}}^{\prime}=2 \frac{\bar{\gamma}_{k} f_{k}\left(x_{k}\right)-f_{k}^{0}}{\left(\bar{\gamma}_{k} f_{k}^{\text {min }}-f_{k}^{0}\right)^{2}} \prod_{i \in K \backslash k} \frac{\left(\bar{\gamma}_{i} f_{i}\left(x_{i}\right)-f_{k}^{0}\right)^{2}}{\left(\bar{\gamma}_{i} f_{i}^{\text {min }}-f_{k}^{0}\right)^{2}}-\lambda=0, k \in K \\
& L_{\lambda}^{\prime}=F-\sum_{k \in K} x_{k}=0 .
\end{aligned}
$$

Eliminating Lagrange multiplier from (11), we get

$$
\frac{\bar{\gamma}_{k} f_{k}\left(x_{k}\right)-f_{k}^{0}}{\left(\bar{\gamma}_{k} f_{k}^{\text {min }}-f_{k}^{0}\right)^{2}} \prod_{i \in K \backslash k} \frac{\left(\bar{\gamma}_{i} f_{i}\left(x_{i}\right)-f_{i}^{0}\right)^{2}}{\left(\bar{\gamma}_{i} f_{i}^{\text {min }}-f_{i}^{0}\right)^{2}}=\frac{\bar{\gamma}_{n} f_{n}\left(x_{n}\right)-f_{n}^{0}}{\left(\bar{\gamma}_{i} f_{n}^{\text {min }}-f_{n}^{0}\right)^{2}} \prod_{i \in K \backslash n} \frac{\left(\bar{\gamma}_{i} f_{i}\left(x_{i}\right)-f_{i}^{0}\right)^{2}}{\left(\bar{\gamma}_{i} f_{i}^{\text {min }}-f_{i}^{0}\right)^{2}}, k, n \in K, n \neq k,
$$

whence we arrive at the system

$$
\left(\bar{\gamma}_{k} f_{k}\left(x_{k}\right)-f_{k}^{0}\right) \prod_{i \in K \backslash k}\left(\bar{\gamma}_{i} f_{i}\left(x_{i}\right)-f_{i}^{0}\right)^{2}=\left(\bar{\gamma}_{n} f_{n}\left(x_{n}\right)-f_{n}^{0}\right) \prod_{i \in K \backslash n}\left(\bar{\gamma}_{i} f_{i}\left(x_{i}\right)-f_{i}^{0}\right)^{2}, k, n \in K, n \neq k,
$$

or

$$
\bar{\gamma}_{k} f_{k}\left(x_{k}\right)-f_{k}^{0}=\bar{\gamma}_{n} f_{n}\left(x_{n}\right)-f_{n}^{0}, n \neq k \in K(13)
$$

Inserting system utility (4) and utility of elements (5) into (13) we arrive at the distribution (10). Differentiating (11), we shall get the system of sufficient conditions of optimality

$L_{x_{k}}^{\prime \prime}=\prod_{i \in K \backslash k} \frac{\left(\bar{\gamma}_{i} f_{i}\left(x_{i}\right)-f_{i}^{0}\right)^{2}}{\left(\bar{\gamma}_{k} f_{i}^{\text {min }}-f_{i}^{0}\right)^{2}}>0, k \in K$,

that are met in case of any $f_{i}\left(x_{i}\right), f_{i}^{0}, f_{i}^{\text {min }}$.

As distribution (10) maximizes the aggregate criterion (9), consequently, in accordance with the results of [10], it is Pareto-efficient.

On the basis of the solution (10) of the multicriteria problem (9) let us lay down the aggregated utility distribution algorithm in the form of the series of the following steps.

1. Input of the given data: functions of criteria $f=\left\{f_{k}(u), k \in K\right\}$ and restrictions de-

fining the ranges of acceptable controls $\mathrm{U}_{\mathrm{k}}$.

2. Determining scalar optimums of criteria (1) and minimums of criteria (2).

3. Determining optimums of criteria in the system (3) and utility of the system 4 (4). 
4. Defining the priorities vector (7).

5. Calculating the utility distribution (10) and the optimal criteria vector (5).

The algorithm realization requires for the criteria feasible region to be not empty $U_{i} \cap U_{k} \neq \varnothing, k \in K \backslash i$ and also for the functions $f=\left\{f_{k}(u), k \in K\right\}$ to have finite maximums in corresponding feasible regions $U_{k}$. These conditions are met in practical problems, because as a rule the regions $U_{k}$, are compact and usually they coincide for different criteria of one system.

\section{$3 \quad$ Utility optimal distributions modelling}

Modelling was done in the context of the three-element system of electronics market, the elements of which are retailer, bank, insurer, with the functions $f_{k}(u), k \in K$ having the form

$f_{k}\left(u_{k}\right)=p_{k} u_{k}^{b_{k}+1}-c_{k} u_{k}, k \in K$,

where $f_{k}, u_{k}, c_{k}$ - k-element utility (profit), output and marginal costs, $p_{k}, b_{k}$-price trends parameters on corresponding markets. Scalar optimums of elements (1) were defined according to the formulas:

$u_{k}^{0}=\left[\frac{c_{k}}{p_{k}\left(b_{k}+1\right)}\right]^{\frac{1}{b_{k}}}, k \in K$.

Parameters of the model, criteria (1) scalar optimums, criteria (2) minimums, criteria (3) system optimums are given in Table 1; system (4) utility equaled F=6989. Calculation of parameters of the model is given $[14,18]$ for the electronics retailer LLC "Eldorado", insurer OJSC "Insurance company "Renaissance"" and bank LLC "Home Credit and Finance Bank", that were assigned indices k=1,2,3 correspondingly. In the considered system element 1 gets maximum utility (utility leader), parameter $u_{1}^{0}$ for which corresponds to the volume of sales of goods and is expressed in physical terms, and utility obtained by elements 2,3 (utility outsiders), are significantly lower. However, their participation is required for the system to be integral. That is why the system utility distribution problem becomes. Outputs $u_{2}^{0}, u_{3}^{0}$ correspond to the volume of insurance and lending and are expressed in monetary terms.

Let us suppose that elements may assign their priorities themselves in the course of integration into the system, following two variants of strategic behaviour.

Firstly, lets consider the variant of non-cooperative game [19], elements wherein don't form coalitions, they assign priorities simultaneously and independently. In this case the Cournot game model [20], symbolized by the letter «K», has the form $\gamma_{k(K)}=\arg \max _{\gamma_{k} \leq 1}\left\{\gamma_{k}\right\}, k \in K$ and leads to the priorities vector $\gamma_{k(K)}=\gamma_{(K)}^{*}, k \in K$. The 
resulting distribution $x_{k(K)}^{*}, k \in K$ (fig. 1) corresponds to the elements equisignificance. In this case the element 1 gets the predominant share of distributed utility, and the share of utility distributed in favour of the elements 2,3 is reduced concurrently with the growth of the equilibrium values of priorities $\gamma_{(K)}^{*}$. Model of game with the institutional leader sensu Stackelberg [21], symbolized by the letter «S», takes the form $\gamma_{k(s)}=\arg \max _{\gamma_{k} \leq 1}\left\{\gamma_{k}\right\}, \gamma_{l(s)}=\arg \max _{\gamma_{k} \leq \gamma_{l} \leq 1} \gamma_{l}, k \in K \backslash l$, where l-element is the leader; the model results in the vector of priorities $\gamma_{k(S)}=\gamma_{(S)}^{*}, \gamma_{l(s)}^{*} \geq \gamma_{(S)}^{*}, k \in K \backslash l$. Distribution $x_{k(S)}^{*}, k \in K$ (fig. 1) shows that the leader sensu Stackelberg (l=3) gets the advantage in spite of being the utility outsider.

Table 1. Parameters of the model and criteria vector

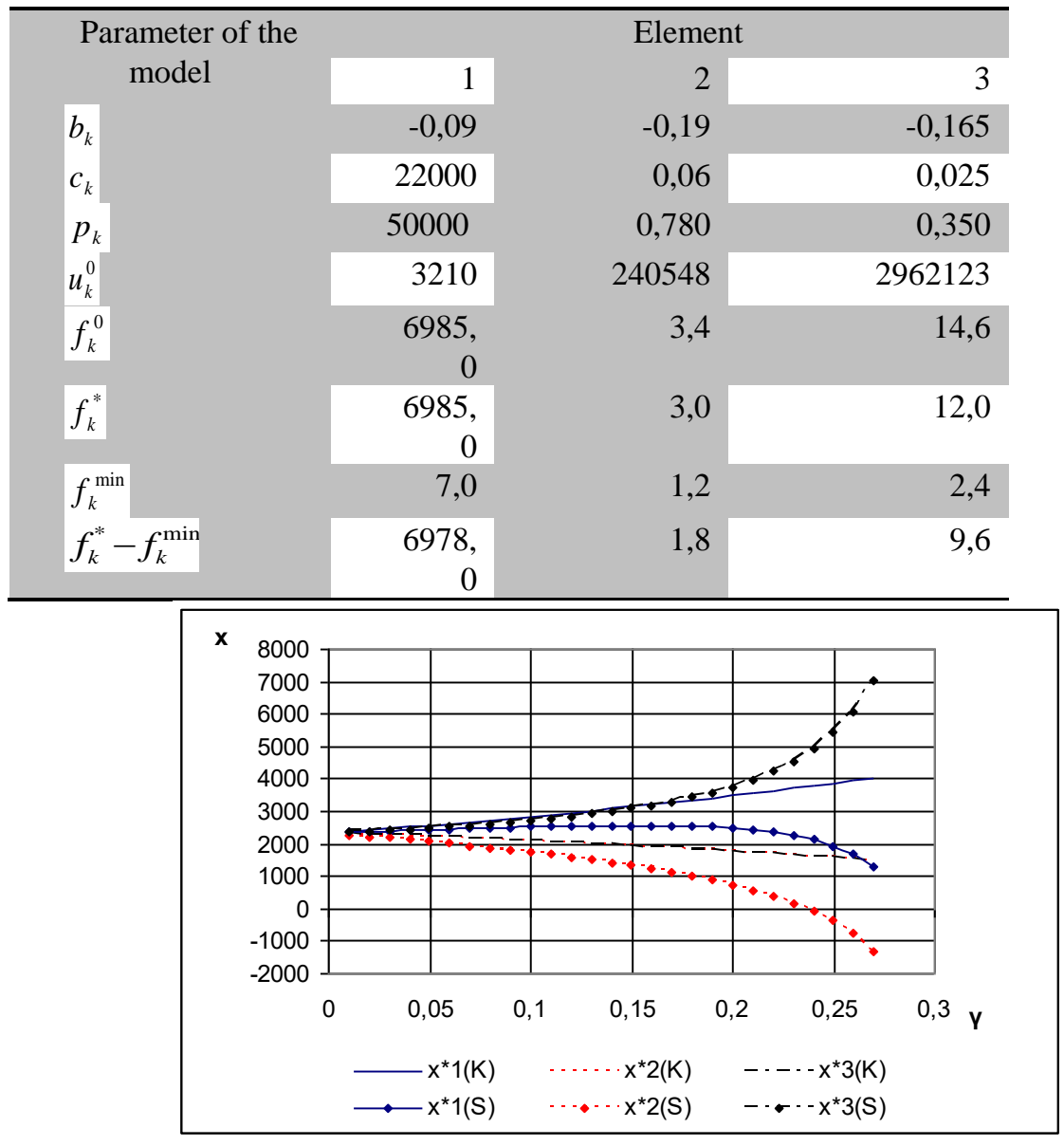

Fig. 1. Utility distribution structure in case of non-cooperative behaviour

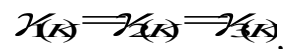

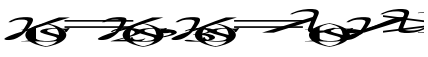


Secondly, let us consider the variant of cooperative game [22] symbolized with the letter "S". In this case elements may form coalitions. The game model of the form $\gamma_{k(C)}=\arg \max _{\gamma_{k} \leq 1}\left\{\gamma_{k}\right\}, \gamma_{l(C)}=\arg \max _{\gamma_{k} \leq \gamma_{l} \leq 1}\left\{\gamma_{l}, l \in L\right\}, k \in K \backslash L$, where index $l \in L$ designates the elements of coalition, leads to the vector of priorities of the form $\gamma_{l(C)}=\gamma_{(C)}^{*}, \gamma_{(C)}^{*} \geq \gamma_{k(C)}^{*}, k \in K \backslash l$. Distribution $x_{k(C)}^{*}, k \in K$ (fig. 2) shows that in comparison with non-cooperative behaviour (fig.1), the coalition of elements $(1,2)$ significantly increases the utility leader's utility and increases the outsider's utility insubstantially. Coalition of utility outsiders $(2,3)$ leads to a drastic utility redistribution in their favour.

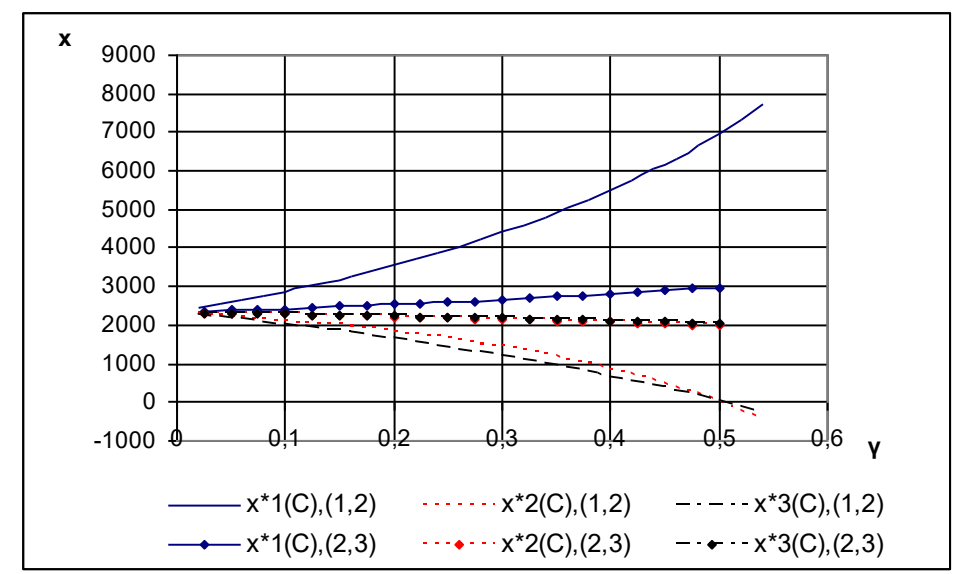

Fig. 2. Utility distribution structure in case of cooperative behaviour

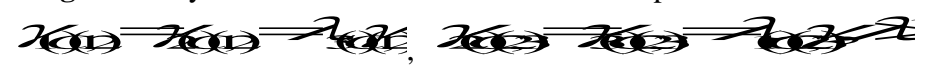

In all the cases examined the system utility was fully distributed between the elements. This confirms Pareto efficiency of distributions that are formed on the basis of the developed algorithm. Modelling showed that the developed algorithm allows to analyze the impact of the priorities vector on the distribution structure and to build the vector of the priorities that express different aims of the system integration.

\section{Conclusion}

The algorithm of solving multicriteria problems of control in strongly coupled systems has been developed. This algorithm allows to determine the optimal system aggregated utility distribution in case of inequisignificant efficiency criteria on the basis of the minimum of deviations of criteria from scalar optimums. The algorithm application sphere includes technical and organizational systems, efficiency criteria of which are quantifiable and preference relations of which have been determined. In case the system elements utilities are transferable, which is in general ensured by the 
criteria normalization. The algorithm is based on the normalized criteria aggregation and in case of the utility function optimization with account taken of priorities, it leads to the minimax distribution (of the guaranteed result). Consequently, the algorithm determines the Pareto-efficient solution of the multicriteria problem.

The use of algorithm allows to reduce the multicriteria problem of optimization in the control space to the set of scalar problems of optimal control and the problem of multicriteria distribution in the criteria space. As the result the unique solution is established, and, moreover, solving of the multicriteria problem is significantly simplified.

\section{References}

1. Nogin VD. Decision making in multicriteria area: numerical approach. Moscow: "Fizmatlit" Publisher, 2005. [in Russian]

2. Neumann G, Morgenstern O. Theory of games and economic behavior. Moscow: "Nauka" Publisher, 1970. [in Russian]

3. Saaty TL. Decision making in functions and feedback. Analitical grids. Moscow: "LKI" Publisher, 2008. [in Russian]

4. Fishhof VG. Goltein BG., Shapiro ZR. Subjective expected utility: decision making model. Procedures for multicriteria evaluation facilities. Moscow: "VPIISI" Publisher, 1984; 9: 24-46. [in Russian]

5. Caverny JP, Bar-Hillel M, Barron FN, Jungerman H. Contributions to decision research. North-Holland, 1993.

6. Smith GR, Speiser F. Logical decision: multimeasure decision analysis software. Golden. CO: PDQ Printing, 1991.

7. Novikov D. Theory of Control in Organizations. New York, Nova Science Publishers, 2013.

8. Roy B. Multy criteria methodology for decision aiding. Dordrecht: Kluwer academic publisher, 1996.

9. Mashunin YK. Methods and models of vector optimization. Moscow: "Nauka" Publisher, 1986. [in Russian]

10. Homenuk VV. Elements of the theory of multi-objective optimization. Moscow: "Nauka" Publisher, 1983 [in Russian]

11. Geraskin MI. Lazarev YN. Terminal Control descent of the spacecraft in the atmosphere and restrictions on movement modes. Proceedings of the Academy of Sciences. Theory and Control Systems. 2001; 5: 168-174.

12. Balakin VL, Geraskin MI, Lazarev YN. Research spacecraft maneuvering capabilities when driving on a suborbital trajectory. Aerospace Engineering and Technology, 1997; 2: 46-48. [in Russian]

13. Geraskin MI. Optimization models of management of hierarchical corporate systems with inter-corporate interactions. Control sciences, 2010; 5: 28-38. [in Russian]

14. Geraskin MI, Manakhov VV. Optimization of interactions in the multi-agent tightly coupled system "retailer - bank - insurer". Control Science, 2015; 4: 9-18. [in Russian]

15. Burkov VN, Gorgidze II, Novikov DA. Models and mechanisms for the distribution of costs and revenues in a market economy. Moscow: Institute of Control Sciences of Russian Academy of Sciences Publisher, 1997. [in Russian] 
16. Burkov VN, Iskakov MB, Korgin NA. Application of generalized median schemes for the construction of the manipulated mechanism multicriterion active expertise. Control sciences, 2008; 4: 38-47. [in Russian]

17. Bondarik VN, Korgin NA. Mechanisms of resource allocation on the basis of manipulated symmetrical anonymous voting procedures with delegation. Control sciences, 2012; 5: 2632. [in Russian]

18. Geraskin MI, Manakhov VV. Analysis of demand curves in the stock and financial markets of monopolistic competition, Actual Problems of Economics and Law, 2016; 2(10): 80-92. [in Russian]

19. Germeyer YuB, Shtilman MS. Non-cooperative repetitive games with arbitrary discounting. DAN Publisher, 1975. [in Russian]

20. Cournot AA. Researches into the Mathematical Principles of the Theory of Wealth. London, Hafner, 1960. Original 1838.

21. Stackelberg H. Market Structure and Equilibrium: 1st Edition. Translation into English, Bazin, Urch \& Hill, Springer, 2011.

22. Aumann RJ. A survey of cooperative games without side payments. Essays in Mathematical Economics, in Honor of Oskar Morgenstern, 2015: 3-27. 